\title{
Whipple's Disease: Diagnostic Value of rpoB Gene PCR from Peripheral Blood Mononuclear Cells
}

\author{
Kathleen Weigt ${ }^{1}$ (I) - Alexandra Wiessner ${ }^{2}$ - Annette Moter $^{2} \cdot$ Florence Fenollar $^{3}$ - Didier Raoult ${ }^{3}$. \\ Kristina Allers ${ }^{1} \cdot$ Thomas Schneider $^{1} \cdot$ Verena Moos $^{1}$
}

Published online: 7 June 2018

(C) Springer International Publishing AG, part of Springer Nature 2018

\begin{abstract}
Introduction Chronic infection with Tropheryma whipplei, known as Whipple's disease (WD), classically affects the gastrointestinal tract, but any organ system may be affected, and isolated manifestations occur. Reliable diagnosis based on a combination of periodic acid-Schiff (PAS) staining, T. whipplei-specific immunohistochemistry (IHC), and polymerase chain reaction (PCR) from duodenal biopsies may be challenging in cases without classical gastrointestinal infection, so the need for additional diagnostic materials is urgent.

Objective Our objective was to evaluate additional diagnostic possibilities for WD.

Methods We analyzed samples from 20 patients with WD and 18 control subjects in a prospective observational pilot study. In addition to WD diagnosis by PAS staining, $T$. whipplei-specific IHC and PCR of duodenal or extra intestinal tissues, whole EDTA blood, peripheral blood mononuclear cells (PBMCs) and PBMC fractions enriched with or depleted of cluster of differentiation (CD)- $14^{+}$cells were examined using T. whipplei rpoB gene PCR.

Results Tropheryma whipplei DNA was detected in 35 of $60(58.3 \%)$ preparations from 16 of 20 patients with WD,
\end{abstract}

Verena Moos

verena.moos@charite.de

1 Department for Gastroenterology, Infectious Diseases, and Rheumatology, Charité-University Medicine Berlin, Campus Benjamin Franklin, Hindenburgdamm 30, 12203 Berlin, Germany

2 Biofilmcenter, German Heart Institute Berlin, Berlin, Germany

3 URMITE, UMR IRD 198/CNRS 6236, Medical Faculty, Mediterranean University, Marseille, France most of whom lacked gastrointestinal signs and characteristic PAS-positive duodenal macrophages.

Conclusion This study provides evidence for the potential suitability of blood, particularly PBMCs, as material to assist in the diagnosis of WD via rpoB gene real-time PCR. Thus, PCR from blood preparations can be helpful for diagnostic decision making in atypical cases of WD.

\section{Key Points}

$R p o B$ gene polymerase chain reaction (PCR) can be an additional tool to assist in the diagnosis of Whipple's disease.

$R p o B$ gene PCR from blood fractions, especially peripheral blood mononuclear cells (PBMCs), enables the surveillance of treatment efficacy and thus appears to have advantages over immunohistochemistry (IHC) and periodic acidSchiff (PAS) staining.

\section{Introduction}

The rare systemic chronic infection with Tropheryma whipplei [1], known as Whipple's disease (WD), has an estimated incidence of 1:1,000,000 [2, 3]. The classical clinical features of WD are polyarthritis, diarrhea, weight loss, and fever [2]. However, as T. whipplei can affect almost any organ, WD cases with non-classical symptoms can be frequent. The standard for diagnosis of WD is 
usually based on periodic acid-Schiff (PAS) staining of macrophages in the duodenal lamina propria [2] and should be confirmed by an independent specific method such as polymerase chain reaction (PCR) [4-7]. However, no systematic data are available concerning the sensitivity or specificity of these routine tests, and no "gold standard" is available. Improved diagnostic methods and clinical awareness means that non-classical WD, often associated with isolated infection in the joints, heart valves, or central nervous system [8-10], is increasingly being recognized $[10,11]$. In cases without the gastrointestinal involvement typical of classical WD (i.e., in the absence of gastrointestinal symptoms and positive PAS staining from the duodenum), confident diagnosis depends on methods that are not routinely available (such as T. whipplei-specific immunohistochemistry [IHC]) or analysis of additional biopsies of the affected organ or heart valve. It appears that PCR and IHC of duodenal tissue can identify more patients and has similar WD detection rates in cases without PASpositive duodenal macrophages $[4,12]$. However, the sensitivity and specificity of PCR and IHC has not been evaluated systematically. Therefore, an additional diagnostic tool that is less invasive would be helpful in inconclusive cases.

Relman et al. [7] proposed the detection of T. whipplei DNA in blood as a promising alternative. However, detection rates using PCR from the whole blood of patients with active WD targeting repeated sequences of $T$. whipplei [4] or from peripheral blood mononuclear cells (PBMC) targeting T. whipplei-specific 16S rDNA [13] have so far been low. These low rates may have been due to the genes targeted for detection, DNA being prepared by methods only optimized for the isolation of eukaryotic DNA, or insufficient amount of material (e.g., $200 \mu \mathrm{l}$ of fluid in Fenollar et al. [4]). Here, we aimed to assess the value of a previously validated more sensitive $r p o B$ gene PCR [14] for the detection of $T$. whipplei DNA in whole blood and PBMCs following DNA isolation optimized for mycobacteria [14]. In addition, given that Raoult et al. [15] reported the immunodetection of $T$. whipplei in circulating monocytes, we evaluated whether the enrichment of cluster of differentiation (CD)- $14^{+}$monocytes is advantageous for the detection of $T$. whipplei DNA.

Therefore, we conducted a prospective observational pilot study to evaluate the relevance of $r p o B$ gene real-time PCR [14] from EDTA blood, purified PBMCs, and PBMC fractions enriched with or depleted of $\mathrm{CD} 14^{+}$monocytes in patients with chronic T. whipplei infection to assist in the diagnosis of WD.

\section{Material and Methods}

\subsection{Patients}

We included 32 patients with symptoms compatible with WD (e.g., treatment-resistant seronegative rheumatoid arthritis, gastrointestinal symptoms) and 18 control subjects with other indications for control gastroscopy from May 2014 until May 2017 (Fig. 1). Cases were identified as previously described [12], with at least two positive results from routine PAS staining, T. whipplei-specific IHC [16]
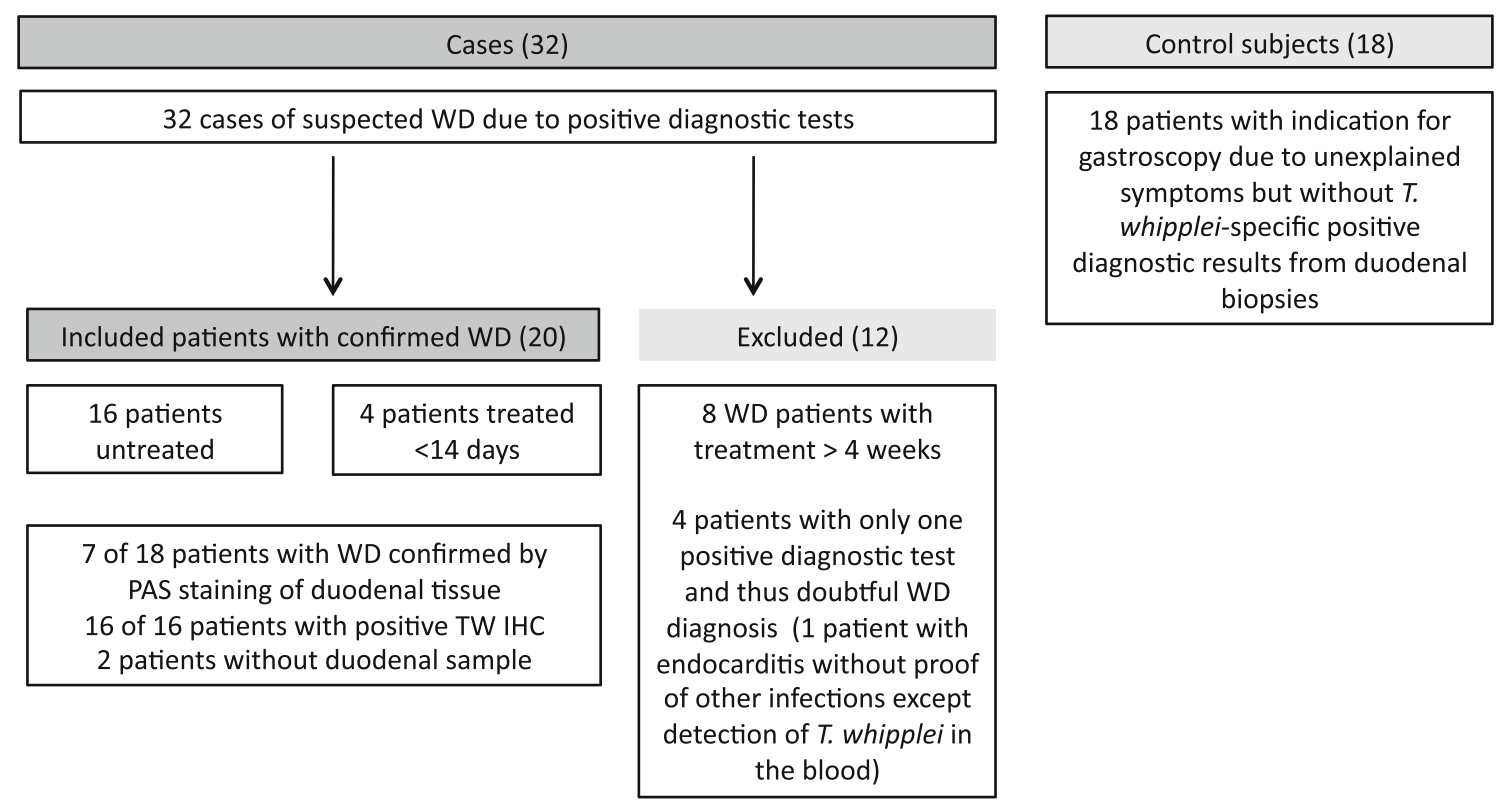

Fig. 1 Details of the analysed cohort with excluded cases, confirmed Whipple disease, and control subjects. WD Whipple's disease, $P A S$ periodic acid-Schiff, PCR polymerase chain reaction, TW IHC T. whipplei-specific immunohistochemistry 
from duodenal or extra intestinal tissues (e.g., lymph node, cardiac valve), or rpoB gene real-time PCR [14] from tissues or fluids (e.g., cerebrospinal fluid, synovial fluid). Every positive $r p o B-\mathrm{PCR}$ result was confirmed by conventional $T$. whipplei-specific $16 \mathrm{~S}$ rRNA amplification based on a slightly modified protocol published by von Herbay et al. [17], using primers TPU5 [18] and whip1 and whip2 [17], subsequent gene sequencing, and comparison with all currently available sequences from public databases (EMBL and GenBank) [14]. Patients with characteristic PAS-positive macrophages in duodenal samples and gastrointestinal signs were defined as having classical WD. We recruited 18 people with indications for gastroscopy but without positive diagnostic tests for T. whipplei as control subjects (Table 1). All control subjects had received a diagnosis other than WD and responded to appropriate treatment; no further indication of WD was found during follow-up (Table 1). A total of 12 patients were excluded because they had only a single positive test, meaning the WD diagnosis was doubtful, or they had received treatment for $>4$ weeks. Among the 20 patients with confirmed WD enrolled in this study (see Table 1 and Fig. 1 for details), 16 were previously untreated and four (patients 12-14 and 17) had received antibiotic treatment up to 14 days before analysis. For six patients (patients 1 , $3,5,10,13$, and 17), follow-up examination was performed 3 months after initiation of antibiotic treatment (see Table 1 for details). All patients and control subjects gave informed consent, and one patient with classical WD (patient 5 , see Table 1) is described in a published case report [19].

\subsection{Sampling of Blood, Isolation of Peripheral Blood Mononuclear Cells (PBMCs) and Sorting of Cluster of Differentiation (CD)-14 ${ }^{+}$ and $\mathrm{CD}^{-}{ }^{-}$PBMCs}

Blood samples were stored at room temperature and processed within $24 \mathrm{~h}$ after sampling. Peripheral blood was collected in EDTA tubes (Vacutainer, BD Biosciences) for whole blood analysis, and PBMCs were isolated from heparinized blood as previously described [20]. CD14 ${ }^{+}$ monocytes were isolated from PBMCs using CD14 MicroBeads (1:10), LS columns, and a MidiMACS magnet according to the manufacturer's protocol (Miltenyi Biotec, Bergisch Gladbach, Germany) [21]. The throughputs of the CD14 MACS sort depleted of CD14 ${ }^{+}$monocytes served as CD14- fractions of PBMCs. Since we hypothesized that $T$. whipplei more reliably persist in $\mathrm{CD} 14^{+}$monocytes, the fraction depleted of $\mathrm{CD} 14^{+}$cells was intended to serve as a kind of negative control with lower detection rates. CD14 ${ }^{+}$ purity of the $\mathrm{CD} 14^{+}$fractions was estimated using flow cytometry with a CD14 (61D3) antibody from eBiocience
(Frankfurt, Germany). While $\mathrm{CD} 14^{+}$fractions of patients with WD $(n=16)$ and of control subjects $(n=11)$ revealed a similar mean percentage of total $\mathrm{CD} 14^{+}$(WD: $91.03 \pm 7.15 \%$; controls: $96.26 \pm 5.67 \%), \mathrm{CD} 14^{\text {high }}$ were significantly lower (WD: $77.75 \pm 16.08 \%$; controls: $94.68 \pm 7.65 \% ; p=0.019)$ and $\mathrm{CD} 14^{\text {low }}$ were significantly higher in patients with WD than in control subjects (WD: $\quad 11.46 \pm 9.10 \%$; $\quad$ controls: $\quad 1.59 \pm 2.04 \%$; $p=0.003) . \mathrm{CD}^{-} 4^{-}$fractions were analyzed for only five patients with WD and revealed a contamination of $8.56 \pm 4.64 \%$ of $\mathrm{CD} 14^{\text {low }}$ and $0.70 \pm 0.44 \%$ of $\mathrm{CD} 14^{\text {high }}$ monocytes.

\subsection{DNA Extraction}

Total genomic DNA was extracted from $1 \mathrm{ml}$ frozen EDTA blood or, corresponding to the cell count of $1 \mathrm{ml}$ blood, from $2 \times 10^{6}$ cells isolated from heparinized blood (PBMCs and cell fractions enriched with or depleted of $\mathrm{CD} 14^{+}$monocytes, respectively). DNA was isolated using the AMPLICOR Respiratory Specimen Preparation Kit (Roche Diagnostics, Mannheim, Germany) according to the manufacturer's instructions, with the following modification for EDTA blood: twice the recommended volume of "respiratory specimen wash solution" was used in a washing step. For all preparations, elution volumes were identical.

To reduce PCR-inhibitory components in DNA extracted from EDTA blood, an additional purification included column-based enrichment via QIAamp DNA Blood Mini Kit (Qiagen, Hilden, Germany).

\subsection{RpoB Gene Real-Time Polymerase Chain Reaction (PCR) Assay}

Total genomic DNA was analyzed for $T$. whipplei DNA by validated $r p o B$ gene real-time PCR as previously described [14]. Primers TwrpoB-F4: CTCGGTGTTGATGTTGATCCAA and TwrpoB-R: GCACCGCAACCTCGGAGAAA [22] were used to amplify a 109-bp segment of the T. whipplei rpoB gene from $5 \mu \mathrm{l}$ of isolated DNA. Realtime detection of the amplicons was achieved using LightCycler hybridization probes TwrpoB-HP1: ACGAGGTCGGATATTATCGC-[FL] $\left(5^{\prime}-3^{\prime}\right)$ and TwrpoB-HP2: [Red 640]-ACAATTCGTTATCTCGCGGCC $\left(5^{\prime}-3^{\prime}\right)$ [14]. Oligonucleotides were synthesized using TIB MOLBIOL (Berlin, Germany), and real-time PCR was performed in a LightCycler instrument, version 2.0 (Roche Diagnostics) as previously described [14].

As standard curve, extracted DNA from a serial dilution of T. whipplei strain Twist ATCC VR-1528 was used, as previously described [14]. The sensitivity of the real-time PCR assay was determined at 17.4 microorganisms per 


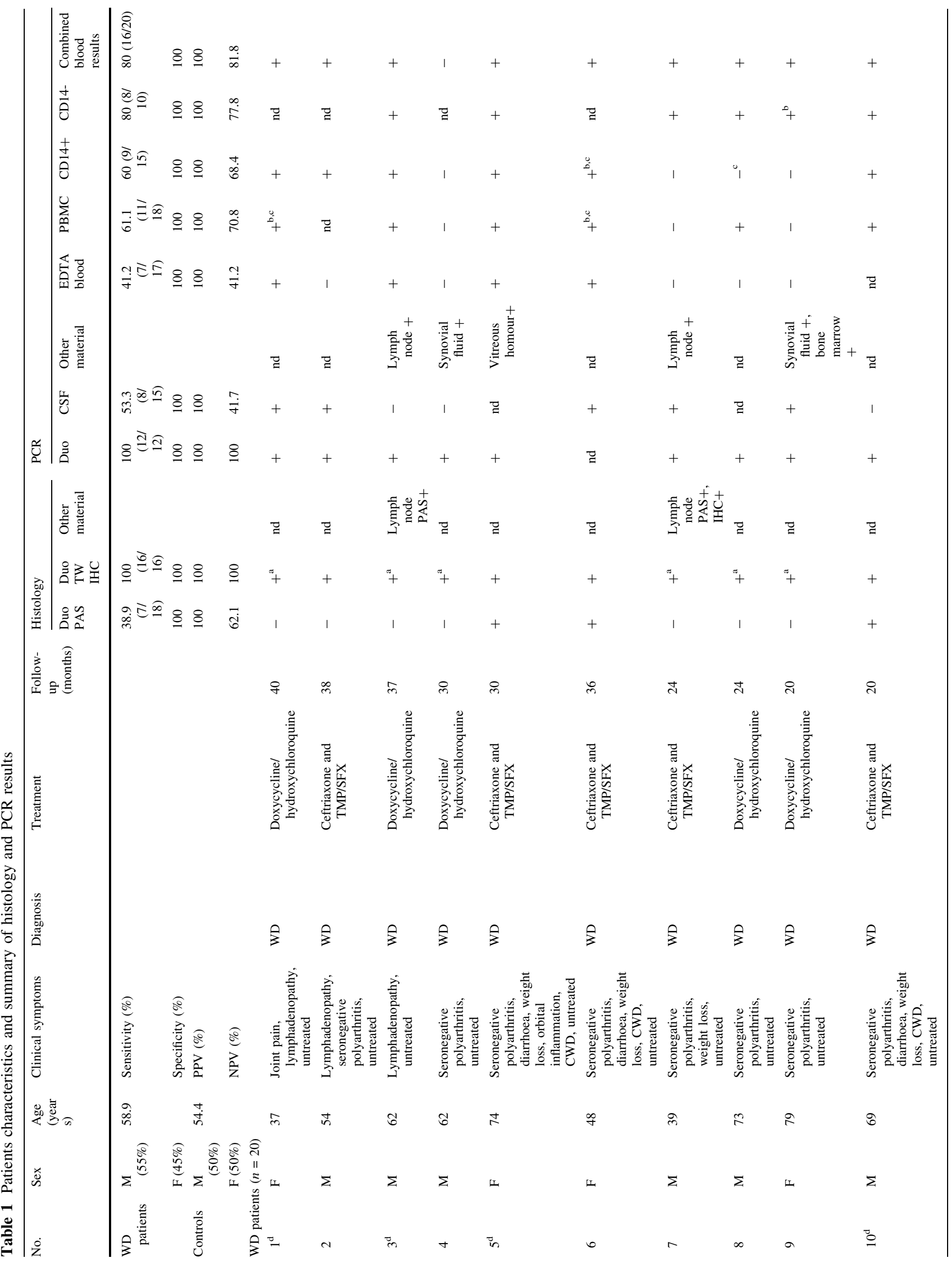




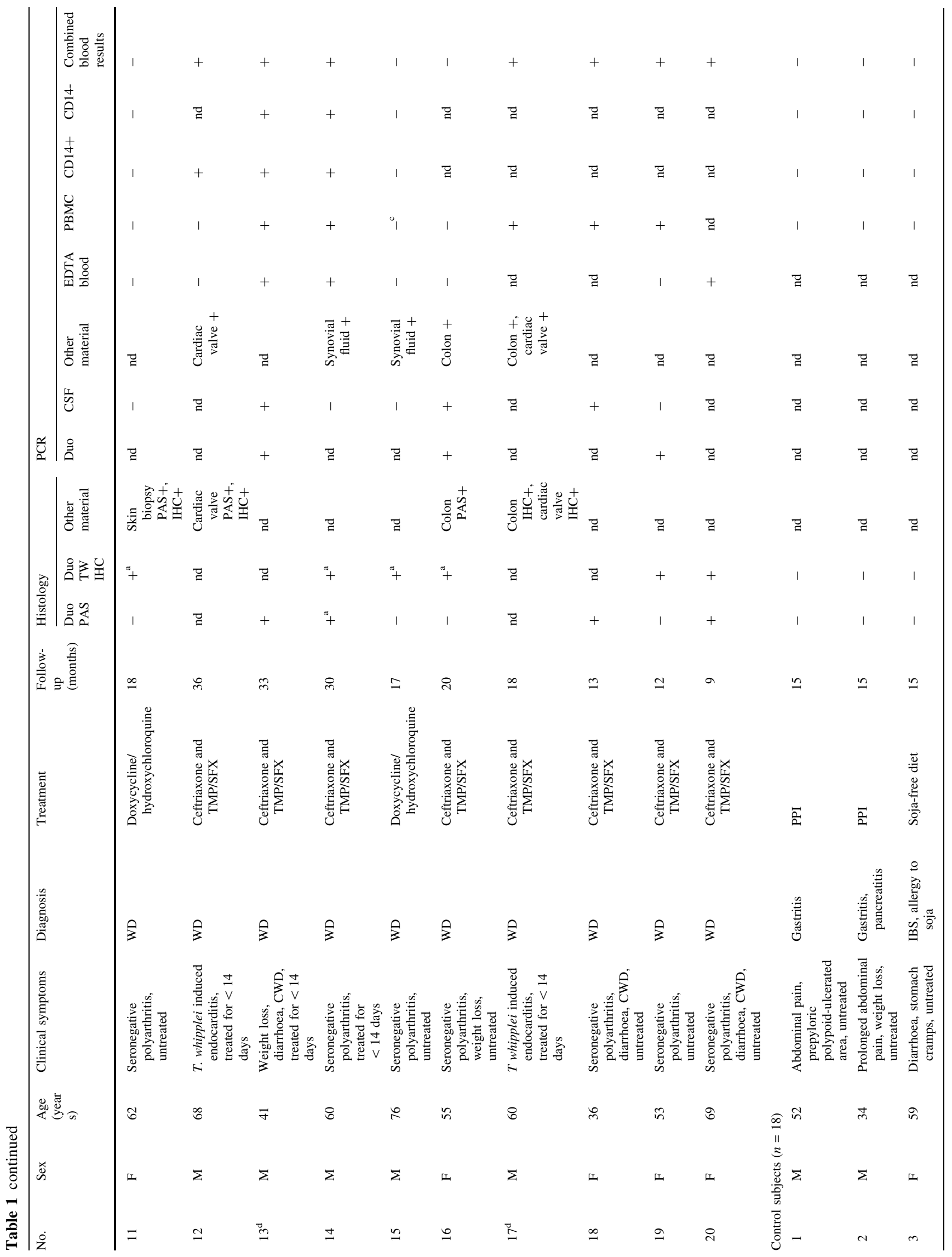




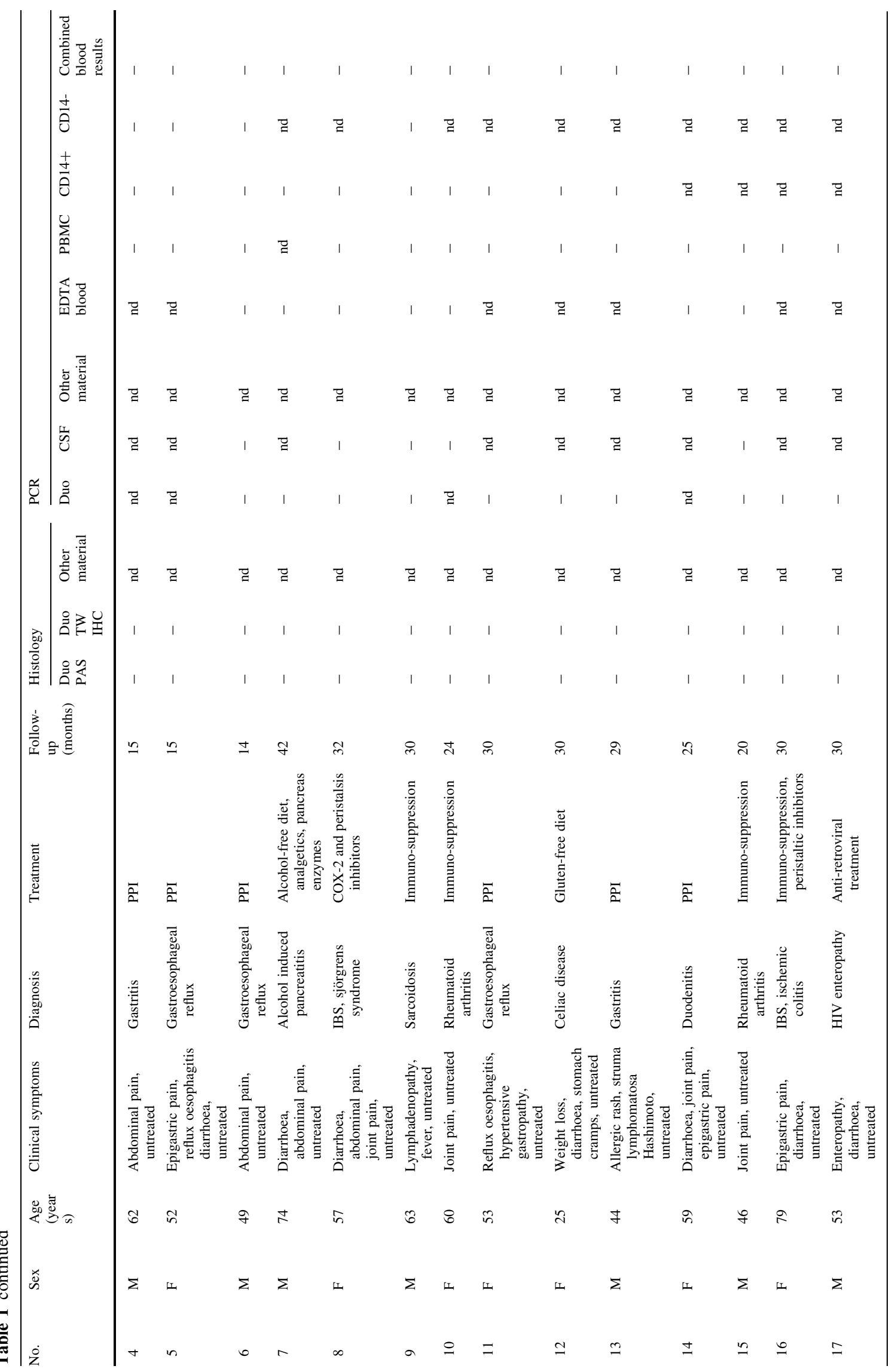




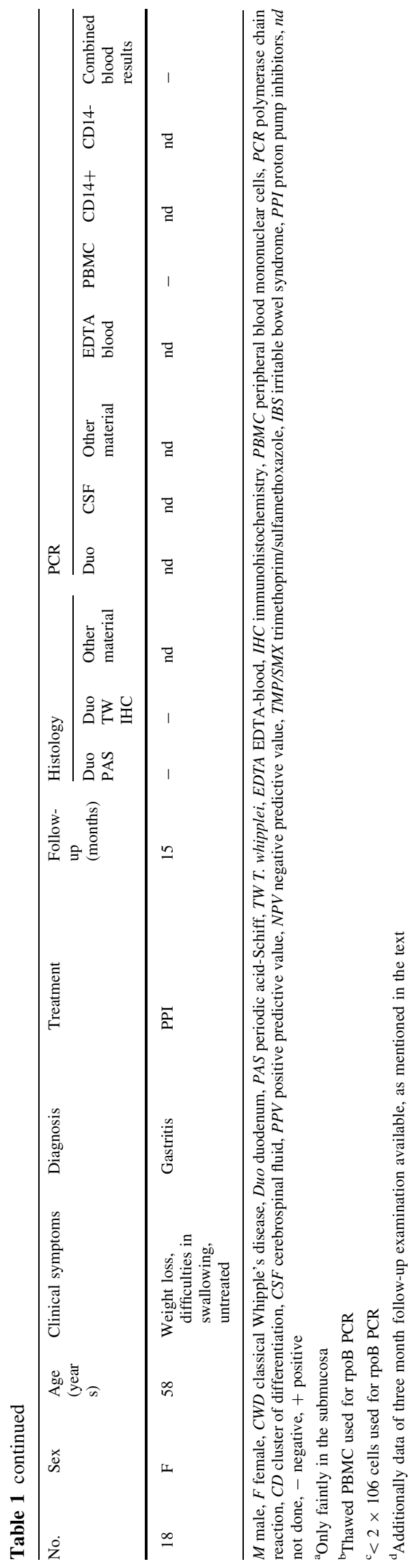

5- $\mu$ l suspension (cycle threshold [CT] $38.0 \pm 0.5$ ). At higher dilutions, the positive results were not consistent in triplicate samples. A high (CT 31.0) and a low (CT 33.0) positive control were included in every PCR run to ensure sensitivity of the actual experiment. To assure optimal amplification in a specific blood sample, an inhibition control at the concentration of the low positive control was also included.

\subsection{Histology and Immunohistochemistry}

Tissue specimens were fixed in formalin and embedded in paraffin, and thin sections were subjected to routine PAS staining. Immunostaining was performed as previously described [16, 23] with rabbit-anti-T. whipplei [16], visualized by donkey-anti-rabbit Biotin (Dianova, Hamburg, Germany), Streptavidin-alkaline phosphatase and Fast red (both DakoCytomation, Glostrup, Denmark). Nuclei were counterstained with Meyer's Hematoxilin (DakoCytomation).

\section{Results}

Among the 20 patients with WD, only six experienced typical clinical symptoms and revealed characteristic PASpositive macrophages in the duodenal lamina propria and were thus defined as having classical WD (Table 1, Fig. 1). One further patient revealed atypical PAS-positive cells in the duodenal submucosa (Table 1, patient 14). Although the specificity of the PAS staining of duodenal biopsies as the classical diagnostic method for WD for our cohort was $100 \%$, the sensitivity (38.9\%) and negative predictive value $(62.1 \%)$ was very low. T. whipplei-specific IHC of the duodenum and rpoB gene PCR from duodenal tissue revealed positive results for all included patients with WD and none of the control subjects (Table 1). Among 11 patients with atypical WD, nine presented with only a faint positive T. whipplei-specific IHC within the duodenal submucosa (Table 1, Fig. 2a, b). Two patients were initially identified via histological analysis of lymph nodes excised to exclude malignant disease (Fig. 2c, d), and two patients with isolated $T$. whipplei-induced endocarditis were diagnosed via analysis of cardiac valves (Table 1, Fig. 2e, f). In addition, PCR from cerebrospinal fluid was positive for 8 of 15 patients (Table 1).

The overall detection rate for T. whipplei in blood samples was 35 of 60 samples and evidenced bacterial DNA in 16 of 20 patients (EDTA: 7/17 patients tested; PBMC: $11 / 18$; enriched for $\mathrm{CD}_{1}{ }^{+}$: $9 / 15$; and depleted of $\mathrm{CD}_{14}^{+}$: 8/10) (Table 1). Upon analysis of the different blood fractions, $r p o B$ gene PCR revealed T. whipplei DNA in EDTA blood of 7 of 17 patients with WD (sensitivity of 
Fig. 2 Immunohistochemical diagnosis of atypical Whipple's disease (WD) from duodenal biopsy (a, b), lymph node (c, d), and heart valve (e, f). Panels demonstrate exemplary presentations of periodic acidSchiff (PAS) staining in purple $(\mathbf{a}, \mathbf{c}, \mathbf{e})$ and Tropheryma whipplei-specific

immunohistochemistry in bright red $(\mathbf{b}, \mathbf{d}, \mathbf{f})$ : a conventional PAS staining of the duodenum reveals no hint of WD; $\mathbf{b} T$. whipplei-specific immunohistochemistry identifies numerous infected cells within the duodenal submucosa; c positive PAS staining in a lymph node with lymphangiectasia (positive areas are marked by arrowheads); d corresponding clearly positive $T$. whippleispecific immunohistochemistry; e inflammatory infiltrate with PAS-positive inclusions in a heart valve (positive areas are marked by arrowheads); $\mathbf{f} T$. whipplei-specific immunohistochemistry demonstrates infection with $T$. whipplei; magnification for all pictures $\times 100$
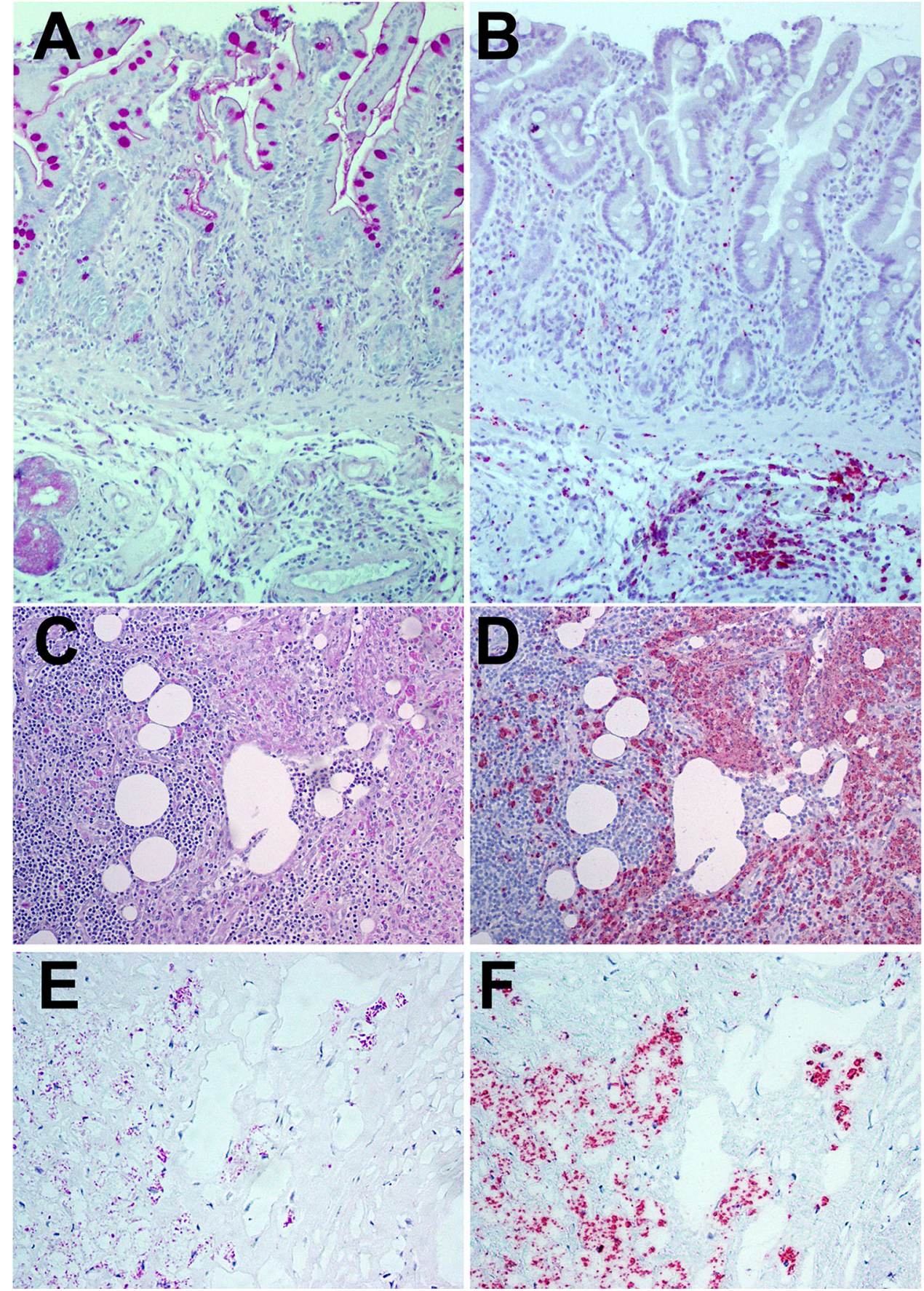

$41.2 \%$ ). The detection rate improved to $61.1 \%$ sensitivity when investigating PBMCs (Table 1). Similar results were obtained investigating $\mathrm{CD} 14^{+}$monocytes. However, the highest sensitivity for T. whipplei DNA (80\%) and the most reliable negative predictive value $(77.8 \%)$ was found in the $\mathrm{CD}_{14}{ }^{+}$-depleted cell fraction (Table 1), originally included for some patients as a negative control.

An interim analysis after inclusion of 15 patients with WD indicated that enrichment of $\mathrm{CD}_{14}{ }^{+}$cells did not improve the $T$. whipplei detection rate compared with
PBMCs, as initially hypothesized. Despite the good results with the $\mathrm{CD} 14^{+}$-depleted PBMCs, we chose PBMCs to assist in WD diagnosis for subsequent patients, resulting in fewer specimens per patient. This decision was based on the lowest CT values for $r p o B$ gene PCR analysing PBMCs, indicating a more reliable level of T. whipplei DNA. While mean CT values from EDTA blood samples were as high as $38.02 \pm 2.02$, the analysis of PBMCs resulted in significantly lower mean $\mathrm{CT}$ values of $32.75 \pm 2.33$ ( $p=0.0016$; Mann-Whitney test). CT values 
of PBMCs enriched for $\mathrm{CD}^{+}(35.22 \pm 2.65)$ and PBMCs depleted of $\mathrm{CD}^{+} 4^{+}(34.76 \pm 3.68)$ were also increased compared with the whole PBMC fraction. Since CT values $>38$ are at the limit of $r p o B$ gene PCR sensitivity [14], high CT values as observed for EDTA blood and $\mathrm{CD} 14^{+}$-depleted and enriched PBMC fractions may produce inconsistent results. In addition, sorting for CD14 is not routine in laboratories, bears a greater risk of contamination, and is associated with additional effort and costs.

Within 14 days of antibiotic treatment, T. whipplei DNA was still detected by $r p o B$ gene PCR (Table 1, patients 12-14, and 17). However, at the 3-month follow-up examination, none of the six specimens tested revealed a positive PCR, indicating a rapid reduction in bacterial DNA in vivo (patients 1, 3, 5, 10, 13, and 17; data not shown). In addition, no $T$. whipplei DNA was detected in any of the 44 blood preparations (EDTA blood, unsorted PBMCs, or PBMCs enriched with or depleted of CD14 ${ }^{+}$) from the 18 control subjects. Therefore, although the sensitivity of the assay depends on the material used for DNA isolation, $r р о B$ gene PCR of blood samples revealed a specificity and a positive predictive value (PPV) of $100 \%$ each in our cohort, confirming this method as a supporting tool in WD diagnosis.

\section{Discussion}

This study has shown the potential suitability of blood and particularly PBMCs as material for $r p o B$ gene real-time PCR to assist in the diagnosis of WD. Awareness of the possibility of isolated WD, atypical—possibly early-WD without gastrointestinal symptoms, and PAS-positive duodenum is increasing $[4,9,10,12]$. Indeed, specific IHC can confirm T. whipplei infection in tissues even in the absence of PAS staining (Fig. 2) [12], but this method is only accessible at specialized centers as the antibodies are not commercialized. PCR from duodenal specimens is also highly indicative of WD [4, 12]; however, healthy luminal carriage of $T$. whipple $i$ without invasive infection may also result in positive PCR [4]. In cases with no gastrointestinal infection, sampling of affected tissue may be virtually impossible. Consequently, alternative diagnostic materials to assure diagnosis by $T$. whipplei-specific PCR from multiple physiologically sterile sites are urgently needed. We demonstrated that detection of $T$. whipplei DNA in whole blood and PBMCs by rpoB gene PCR seems to be highly indicative of WD, even in the absence of intestinal infection and duodenal PAS staining. In our cohort, PAS staining of duodenal biopsies identified only $38.9 \%$ of patients, whereas the combined analysis of PBMCs and PBMC fractions enriched with or depleted of $\mathrm{CD} 14^{+}$ monocytes enabled detection of bacterial DNA in 16 of 20 patients with confirmed WD $(80 \%)$. The feasibility of PCR with PBMCs from patients following short-term treatment was demonstrated by positive results in four patients with WD (two with isolated T. whipplei-induced endocarditis) who received appropriate antibiotic treatment for up to 2 weeks prior to blood analysis.

PCR from PBMCs identified more patients, possibly indicating a higher bacterial load, and revealed an enrichment of T. whipplei DNA, indicated by lower CT values as compared with PCR from EDTA blood, and thus seems superior. However, detection of T. whipplei DNA did not increase after enrichment of $\mathrm{CD}^{+} 4^{+}$monocytes, and $T$. whipplei DNA was also detected in PBMC depleted of $\mathrm{CD}^{+} 4^{+}$monocytes. Even though the PBMC fraction depleted for $\mathrm{CD}_{14}{ }^{+}$cells exhibited the best diagnostic results in our cohort, we recommend PBMCs as the most suitable material to assist in the diagnosis of WD. PBMCs enabled a higher detection rate at lower CT values of PCR compared with EDTA blood, and the depletion of CD14 ${ }^{+}$ monocytes necessitates an intensive work-up of the material, which carries a greater risk of contamination. In addition, $\mathrm{CD} 14^{+}$depletion might be difficult to introduce into routine diagnostic laboratories.

Obvious advantages of using PBMCs to assist in the diagnosis of WD include the simple accessibility of blood, the feasibility for outpatients, and the ability to standardize sampling and cell numbers for semi-quantitative PCR results. The $r p o B$ real-time PCR sensitivity was determined for approximately 17 target organisms per $5 \mu \mathrm{l}$ of $T$. whipplei culture used for DNA extraction (3480 organisms/ ml) [14]. Although the rpoB real-time PCR on PBMCs has not yet been validated, we have already found that $2 \times 10^{6}$ PBMC - a comparatively low number-enabled the detection of $T$. whipplei. On the basis of our experience, $2 \times 10^{6}$ cells correspond to the average amount of PBMCs in $1 \mathrm{ml}$ of peripheral blood of healthy donors. Thus, minimal blood is needed for T. whipplei screening, which is an advantage for patients with diminished health. Another benefit of $r p o B$ gene PCR from PBMCs could be the rapid clearance of $T$. whipplei DNA after initiation of treatment, which — unlike PAS and IHC of duodenal specimens-may allow monitoring of treatment efficacy or possibly early detection of relapse. The lack of benefit from enriching $\mathrm{CD}_{14}{ }^{+}$monocytes for detection of $T$. whipplei DNA hints at a small proportion of infected monocytes only and suggests that $T$. whipplei are not necessarily only associated with classical CD14 ${ }^{\text {high }}$ monocytes, as previously presumed, but also with other cells. Classical CD14 ${ }^{\text {high }}$ monocytes have been demonstrated to eliminate $T$. whipplei in vitro [24]; thus, T. whipplei DNA might have been degraded more effectively in the PBMC fractions enriched for $\mathrm{CD}^{+} 4^{+}$monocytes. In contrast, the persistence of $T$. 
whipplei in monocytes is enabled by interleukin (IL)-16 and has been demonstrated to be associated with an upregulation of CD16 [24]. CD16 is only expressed on non-classical $\mathrm{CD} 14^{\text {low }}$ monocytes, which appear to be elevated during acute infection [25]. The percentage of $\mathrm{CD} 14^{\text {high }}$ monocytes in the $\mathrm{CD} 14^{+}$monocyte fractions of patients with WD was significantly lower as compared with controls (77.75\% in WD patients versus $94.68 \%$ in controls). However, non-classical $\mathrm{CD} 14^{\text {low }}$ monocytes were significantly enhanced in the $\mathrm{CD}_{1}{ }^{+}$fraction of patients with WD; when they were included in the analysis, the purity of the $\mathrm{CD} 14^{+}$fractions was similar for patients and controls. The non-classical CD $14^{\text {low }}$ monocytes in the untreated patients with WD included in our cohort might be activated by the pathogen itself or by enhanced lipopolysaccharides in the serum [26]. Thus, the high percentage of $\mathrm{CD} 14^{\text {low }}$ monocytes in patients with WD might be more difficult to access for the antibodies used for depletion, resulting in contamination of the CD14-depleted fraction with $\mathrm{CD} 14^{\text {low }}$ non-classical monocytes that might be preferentially loaded with $T$. whipplei.

As classical CD14 ${ }^{\text {high }}$ monocytes and dendritic cells were both removed from the PBMC fraction by depleting $\mathrm{CD}_{14}{ }^{+}$cells, T. whipplei-positive cells are potentially lymphocytes [27], $\mathrm{CD} 16^{+} \mathrm{CD} 14^{\text {low }}$ non-classical monocytes [24], or basophilic granulocytes. The variable positive results in different cell preparations for some patients possibly indicates day-to-day variability in blood samples or a low level of infected cells in the preparations.

The rроB gene PCR recognized T. whipplei DNA in PBMCs and enriched $\mathrm{CD} 14^{+}$monocytes, respectively, from two patients with $T$. whipplei-induced endocarditis (Table 1, patients 12 and 17, diagnosed from excised heart valve). One additional patient with endocarditis revealed positive PCR results from PBMCs but was excluded because heart valve replacement was not conducted, meaning a second diagnostic finding to confirm WD was not possible (see Fig. 1). Thus, this study indicates rроB gene PCR for the detection of $T$. whipplei DNA in isolated PBMCs is a promising non-invasive material for decision making in the diagnosis of WD, especially for atypical WD when sampling of infected tissue is challenging (e.g., heart valves).

Further studies to determine diagnostic benefits should include more patients with isolated T. whipplei endocarditis, healthy patients with T. whipplei in stool samples, and recurrent testing of blood samples and greater cell numbers to determine the intraindividual variability of the results. Importantly, identification of cell populations associated with $T$. whipplei is of particular interest to understand the pathways of the pathogen in the human body. Until further studies evaluate these issues, this approach should be restricted to specialized laboratories for inconclusive cases.

\section{Compliance with Ethical Standards}

Funding This work was supported by Deutsche Forschungsgemeinschaft SFB633, KFO104, SCHN 616/6-2, European commission QLG1-CT-2002-01049 and Charité doctorate grants. The German Consiliary Laboratory for T. whipplei is supported by the Robert Koch Institute. The funders had no role in the study design, the data collection and interpretation, or the decision to submit the work for publication.

Conflict of interest KW, AW, AM, FF, DR, KA, TS, and VM have no conflicts of interest.

Informed consent Informed consent was obtained from all individual participants included in the study (ethical commission of the Charité; EA4/122/10).

\section{References}

1. Fenollar F, Puechal X, Raoult D. Whipple's disease. N Engl J Med. 2007;356(1):55-66.

2. Schneider T, Moos V, Loddenkemper C, Marth T, Fenollar F, Raoult D. Whipple's disease: new aspects of pathogenesis and treatment. Lancet Infect Dis. 2008;8(3):179-90.

3. La Scola B, Fenollar F, Fournier PE, Altwegg M, Mallet MN, Raoult D. Description of Tropheryma whipplei gen. nov., sp. nov., the Whipple's disease bacillus. Int J Syst Evol Microbiol. 2001;51(Pt 4):1471-9.

4. Fenollar F, Laouira S, Lepidi H, Rolain JM, Raoult D. Value of Tropheryma whipplei quantitative polymerase chain reaction assay for the diagnosis of Whipple disease: usefulness of saliva and stool specimens for first-line screening. Clin Infect Dis. 2008;47(5):659-67. https://doi.org/10.1086/590559.

5. Hinrikson HP, Dutly F, Nair S, Altwegg M. Detection of three different types of 'Tropheryma whippelii' directly from clinical specimens by sequencing, single-strand conformation polymorphism (SSCP) analysis and type-specific PCR of their 16S-23S ribosomal intergenic spacer region. Int $J$ Syst Bacteriol. 1999;4:1701-6.

6. Moos V, Schneider T. Changing paradigms in Whipple's disease and infection with Tropheryma whipplei. Eur J Clin Microbiol Infect Dis. 2011;30(10):1151-8.

7. Relman DA, Schmidt TM, MacDermott RP, Falkow S. Identification of the uncultured bacillus of Whipple's disease. N Engl J Med. 1992;327(5):293-301.

8. Fenollar F, Celard M, Lagier JC, Lepidi H, Fournier PE, Raoult D. Tropheryma whipplei endocarditis. Emerg Infect Dis. 2013;19(11):1721-30.

9. Geissdorfer W, Moos V, Moter A, Loddenkemper C, Jansen A, Tandler R, et al. High frequency of Tropheryma whipplei in culture-negative endocarditis. J Clin Microbiol. 2012;50(2):216-22.

10. Lehmann P, Ehrenstein B, Hartung W, Dragonas C, Reischl U, Fleck M. PCR analysis is superior to histology for diagnosis of Whipple's disease mimicking seronegative rheumatic diseases. Scand J Rheumatol. 2016. https://doi.org/10.1080/03009742. 2016.1183038.

11. Lagier JC, Fenollar F, Raoult D. Whipple's disease and Tropheryma whipplei infections in internal medicine. When to think about it? How to treat? La Revue de medecine interne/fondee par 
la Societe nationale francaise de medecine interne. 2014;35(12):801-7. https://doi.org/10.1016/j.revmed.2014.04. 016.

12. Gunther U, Moos V, Offenmuller G, Oelkers G, Heise W, Moter A, et al. Gastrointestinal diagnosis of classical Whipple disease: clinical, endoscopic, and histopathologic features in 191 patients. Medicine. 2015;94(15):714.

13. Marth T, Fredericks D, Strober W, Relman DA. Limited role for PCR-based diagnosis of Whipple's disease from peripheral blood mononuclear cells. Lancet. 1996;348(9019):66-7.

14. Moter A, Schmiedel D, Petrich A, Wiessner A, Kikhney J, Schneider $\mathrm{T}$, et al. Validation of an rpoB gene PCR assay for detection of Tropheryma whipplei: 10 years' experience in a National Reference Laboratory. J Clin Microbiol. 2013;51(11):3858-61. https://doi.org/10.1128/JCM.01703-13.

15. Raoult D, Lepidi H, Harle JR. Tropheryma whipplei circulating in blood monocytes. N Engl J Med. 2001;345(7):548.

16. Lepidi H, Costedoat N, Piette JC, Harle JR, Raoult D. Immunohistological detection of Tropheryma whipplei (Whipple bacillus) in lymph nodes. Am J Med. 2002;113(4):334-6.

17. von Herbay A, Ditton HJ, Maiwald M. Diagnostic application of a polymerase chain reaction assay for the Whipple's disease bacterium to intestinal biopsies. Gastroenterology. 1996;110(6):1735-43.

18. Lane DJ, Pace B, Olsen GJ, Stahl DA, Sogin ML, Pace NR. Rapid determination of 16S ribosomal RNA sequences for phylogenetic analyses. Proc Natl Acad Sci USA. 1985;82(20):6955-9.

19. Blessin UB, Fischer A, Schneider T, Moos V, Muller T, Weylandt KH, et al. More than meets the eye. Gut. 2018;67(1):69. https://doi.org/10.1136/gutjnl-2016-312390.
20. Schinnerling K, Moos V, Geelhaar A, Allers K, Loddenkemper $\mathrm{C}$, Friebel J, et al. Regulatory $\mathrm{T}$ cells in patients with Whipple's disease. J Immunol. 2011;187(8):4061-7. https://doi.org/10.4049/ jimmunol.1101349.

21. Schinnerling K, Geelhaar-Karsch A, Allers K, Friebel J, Conrad $\mathrm{K}$, Loddenkemper $\mathrm{C}$, et al. Role of dendritic cells in the pathogenesis of Whipple's disease. Infect Immun. 2015;83(2):482-91.

22. Drancourt M, Carlioz A, Raoult D. rpoB sequence analysis of cultured Tropheryma whippelii. J Clin Microbiol. 2001;39(7):2425-30. https://doi.org/10.1128/JCM.39.7.24252430.2001.

23. Moos V, Schmidt C, Geelhaar A, Kunkel D, Allers K, Schinnerling $\mathrm{K}$, et al. Impaired immune functions of monocytes and macrophages in Whipple's disease. Gastroenterology. 2010;138(1):210-20. https://doi.org/10.1053/j.gastro.2009.07. 066.

24. Desnues B, Raoult D, Mege JL. IL-16 is critical for Tropheryma whipplei replication in Whipple's disease. J Immunol. 2005;175(7):4575-82.

25. Strauss-Ayali D, Conrad SM, Mosser DM. Monocyte subpopulations and their differentiation patterns during infection. J Leukoc Biol. 2007;82(2):244-52. https://doi.org/10.1189/jlb. 0307191.

26. Epple HJ, Friebel J, Moos V, Troeger H, Krug SM, Allers K, et al. Architectural and functional alterations of the small intestinal mucosa in classical Whipple's disease. Mucosal Immunol. 2017;10(6):1542-52. https://doi.org/10.1038/mi.2017. 6.

27. Fredricks DN, Relman DA. Localization of Tropheryma whippelii rRNA in tissues from patients with Whipple's disease. J Infect Dis. 2001;183(8):1229-37. 